\title{
DIRECT SIMULATIONS OF THE QUANTUM MODEL FOR $\mathrm{CsNiF}_{3}$
}

\author{
A. Caramico D'Auria, F. Esposito, U. Esposito \\ Dipartimento di Scienze Fisiche, Universitá di Napoli, Piazzale Tecchio, Napoli, Italy \\ G. Kamieniarz* and R. Dekeyser \\ Institute of Theoretical Physics, University of Leuven \\ Celestijnenlaan 200D, Leuven, Belgium
}

\begin{abstract}
We address the problem of reliability of a finite-chain technique for $\mathrm{CsNiF}_{3}$. We investigate the effect of the boundary conditions, completely neglected so far, and apply a new extrapolation procedure appropriate for quantities showing non-monotonic behaviour. From a detailed analysis of the specific heat existing theoretical estimates for the model parameters are discriminated and a strong evidence for the reliability of the direct finite-chain technique predictions is presented.
\end{abstract}

PACS numbers: $64.60 . \mathrm{Cn}, 75.10 . \mathrm{Dg}, 75.10 . \mathrm{Jm}, 75.40 . \mathrm{Cx}, 75.40 . \mathrm{Mg}$

\section{Description}

The numerical finite-size technique has been applied here to study thermodynamic properties of the $S=1$ quasi-one-dimensional ferromagnet $\mathrm{CsNiF}_{3}$ which can be described by the spin Hamiltonian

$$
\mathcal{H}=-J \sum_{i=1}^{N-1} S_{i} \cdot S_{i+1}+A \sum_{i=1}^{N}\left(S_{i}^{z}\right)^{2}-g \mu_{B} B \sum_{i=1}^{N} S_{i}^{\alpha},
$$

where $J$ denotes the ferromagnetic $\mathrm{CsNiCl}_{3}$ coupling constant, $A$ - the anisotropy parameter and $B$ - the external magnetic field which can be applied in the chain direction $(\alpha=z)$ or in the easy plane $(\alpha=x)$.

It is well known that, under certain conditions, the equation of motion of the spin chain described by the easy plane Heisenberg model with the in-plane field can be mapped onto a classical sine-Gordon equation yielding linear and non-linear excitations. Due to the lack of exact descriptions and to some discrepancies, numerical simulations of the corresponding spin models have been performed both for statics and dynamics ([1, 2] and ref. therein).

*Permanent address: Institute of Physics, A. Mickiewicz University, Matejki 48/49, 60-769 Poznań, Poland. 
After having diagonalized the matrix representation of the Hamiltonian with free boundary conditions, we evaluate the corresponding thermodynamic quantities for $2 \leq N \leq N_{\max }$ and we perform linear extrapolations in $1 / N$. The error bars and the uncertainties have been estimated taking into account the variations of the extrapolated values with respect to the number of points as well as new parabolic extrapolations.

First, we briefly recall our previous results [1]. Extending the length of the chains $(N \leq 8)$, we calculated the zero-field specific heat $C$, the parallel susceptibility $\chi_{\|}$and the perpendicular susceptibility $\chi_{\perp}$, and we fitted them to the available zero-field experimental data. The $g$-factors for $\mathrm{CsNiF}_{3}$ are anisotropic [3] and have the following values: $g_{\|}=2.23, g_{\perp}=2.28$, where $\|$ and $\perp$ stand for the field directions with respect to the chain axis.

Assuming the $g$ values as quoted above, the best fit was reached for

$$
J / k_{\mathrm{B}}=20.5 \pm 1.0 \mathrm{~K}, \quad A / J=0.425 \pm 0.15 \text {. }
$$

As to the field-dependent behaviour, we found in the excess specific heat

$$
\Delta C(T, B)=C(T, B)-C(T, 0)
$$

well defined maxima, showing quantitative agreement with experiments. A comparison with the magnetization measurements in the hard direction and the FMR observations of the field dependence of the spin-wave energy gap at $q=0$ revealed an overall agreement with experiment for our choice of the parameters $J$ and $A$ [1].

\section{Results}

In the remainder of this paper we address the problem of the reliability of our calculations. In order to avoid systematic errors related to the extrapolation procedure:

1. we computed the zero-field specific heat for sizes up to $N=9$;

2. we modified the boundary conditions;

3. we adapted a new extrapolation procedure, locating first the position and height of the peaks in the zero-field specific heat and then the parts of the curves around the maxima.

Our new data for the specific heat (extracted from system sizes $N \leq 9$ ) are presented in Table I, according to the choice of parameters displayed in the first row and taken after $[1,2,4]$. Within quoted error bars we do not find deviations with respect to our earlier results (Fig. 12 in [1]. Note that some parameters in [1] are slightly different from those considered here.)

As to the boundary conditions (bc), we analyzed two cases. We imposed the periodic $b c$ and we applied the free $b c$, reducing the border single-ion anisotropy term by a factor of 2 so that our Hamiltonian can be considered as a sum over the pair of interactions. We found that the periodic bc significantly deteriorate the convergence in the low temperature regime and we could not confirm our previous estimates, except for high temperatures. However, the latter modification of the bc, changing the finite-size results, does not affect the convergence and the extrapolated data are left invariant within an error margin. An example of these calculations is shown in Fig. 1. 
The extrapolated zero-field specific heat data from

\section{TABLE I}

system sizes $N \leq 9$ in molar units. The uncertainties

in the last decimal place are given in parentheses.

\begin{tabular}{c|c|c|c}
\hline \hline$T$ & $J / k_{\mathrm{B}}=20.5 \mathrm{~K}$ & $J / k_{\mathrm{B}}=25.0 \mathrm{~K}$ & $J / k_{\mathrm{B}}=23.6 \mathrm{~K}$ \\
\hline 4.0 & $1.99(1)$ & $2.82(1)$ & $1.80(1)$ \\
5.0 & $2.410(5)$ & $2.20(1)$ & $2.21(1)$ \\
7.0 & $2.887(1)$ & $2.614(4)$ & $2.724(3)$ \\
9.0 & $2.9885(2)$ & $2.7079(5)$ & $8806(3)$
\end{tabular}

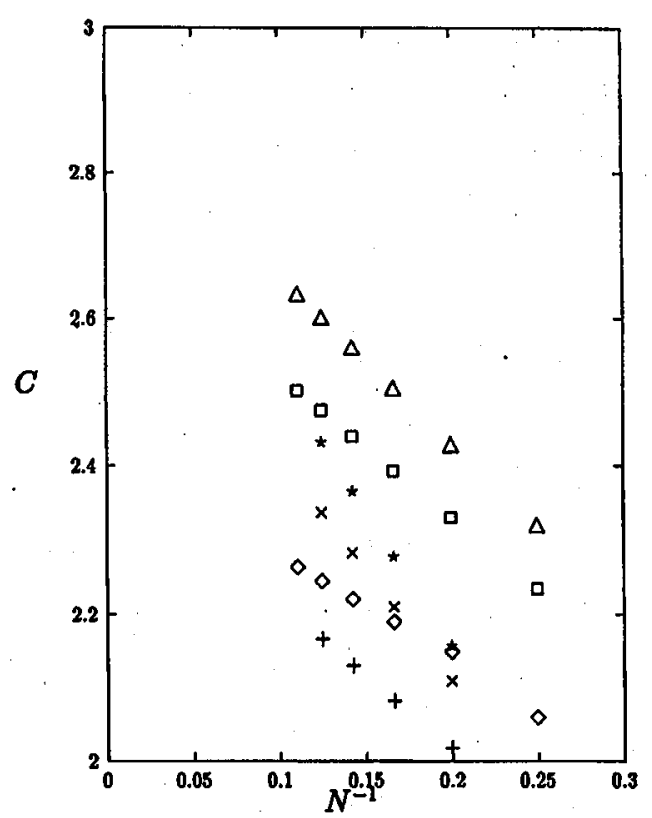

Fig. 1. The zero-field specific heat of the finite chains in units $[\mathrm{J} / \mathrm{mole} \mathrm{K}]$. The symbols $\diamond, \square$ and $\Delta$ report the finite-size results at $T=5,6$ and $7 \mathrm{~K}$, respectively. The remaining symbols $+, x, \star$ show the size dependence for the modified boundary conditions as described in the text.

We also tested our estimates, changing the extrapolation procedure. So far the extrapolations have been performed for the finite-size data at a given temperature. We modify this, looking for maxima $C_{N}^{\max }$ in the specific heat for each finite $N$ which is found in different temperatures $T_{N}^{\max }$. Next, we extrapolate our new data for the maxima. We recover the values calculated before as shown in Table II. Similar calculations in the vicinity of the maxima reproduce within error bars the estimates from the finite-size isotherms (Fig. 12 in [1] and Table I given here).

Summarizing, our present results indicate the reliability of our finite-size 
TABLE II

The finite-size values $C_{N}^{\max }$ and the extrapolated maxima of the specific heat in molar units.

\begin{tabular}{c|c|c}
\hline \hline$N$ & $J / k_{\mathrm{B}}=20.5 \mathrm{~K}$ & $J / k_{\mathrm{B}}=23.6 \mathrm{~K}$ \\
\hline 5 & 2.483008 & 2.374206 \\
6 & 2.567178 & 2.454407 \\
7 & 2.627269 & 2.515650 \\
8 & 2.672414 & 2.562320 \\
9 & 2.707559 & 2.598809 \\
$\infty$ & $2.989(1)$ & $2.89(1)$
\end{tabular}

calculations, give a new evidence in favour of the parameters fixed in [1] and prompt new zero-field specific heat measurements to verify our predictions.

\section{References}

[1] L.S. Campana, A. Caramico D'Auria, F. Esposito, U. Esposito, R.W. Gerling, G. Kamieniarz, J. Phys., Condens. Matter 4, 2915 (1992).

[2] T. Delica, W.J.M. de Jonge, K. Kopinga, H. Leschke, H.J. Mikeska, Phys. Rev. B 44, 11773 (1991).

[3] C. Dupas, J.P. Renard, J. Phys. C 10, 5057 (1977).

[4] A. Cuccoli, V. Tognetti, P. Verrucchi, R. Vaia, Phys. Rev. B 44, 903 (1991). 